\title{
LOS RAYOS CÓSMICOS DE LA UNIVERSIDAD DE ALCALÁ
}

\section{COSMIC RAYS AT THE UNIVERSITY OF ALCALÁ}

Ruth Parra González: Universidad de Alcalá de Henares. Madrid (España) ruth.parra@uah.es

\section{CURRÍCULUM VITAE}

Coordinadora del Área de Cultura Científica de la OTRI de la Universidad de Alcalá de Henares (España). Coordinadora del Programa Ciencia y Sociedad Sistema Madrid - Alcalá de Henares (España)

\section{RESUMEN}

La Universidad de Alcalá de Henares participa en el proyecto del mayor observatorio de rayos cósmicos del mundo. El observatorio, que lleva el nombre de Pierre Auger Sur, se inauguró el pasado 9 de noviembre, coincidiendo con el Año Internacional de la Física. Los integrantes del Grupo de Plasmas Espaciales y Astropartículas (SPA) de la Universidad de Alcalá colaboran en este importante proyecto internacional. En este artículo se explica el mismo, incluyendo además varias ilustraciones.

\section{PALABRAS CLAVE}

Observatorio - Rayos cósmicos - Proyecto - Universidad de Alcalá 


\section{ABSTRACT}

The University of Alcalá de Henares involved in the project's largest cosmic ray observatory in the world. The observatory, named after Pierre Auger South, was opened on 9 November, coinciding with the International Year of Physics. The members of the Group of Space Plasmas and Astroparticle (SPA) at the University of Alcalá collaborate in this important international project. This article explains the same, also including several illustrations.

\section{KEY WORDS}

Observatory - Cosmic rays - Project - University of Alcalá

\section{TEXTO:}

Coincidiendo con el Año Internacional de la Física, la comunidad científica mundial colabora, de nuevo, en un proyecto de gran envergadura que reúne más de 300 científicos, pertenecientes a 55 instituciones de 16 países. En realidad trabajan en el observatorio físicos de 19 nacionalidades, pues algunos de ellos participan a título individual, sin contar con el apoyo oficial de sus gobiernos.

Los integrantes del Grupo de Plasmas Espaciales y Astropartículas (SPA) de la Universidad de Alcalá, encabezados por Julio Gutiérrez, Catedrático de Física Atómica, Molecular y Nuclear, Luis del Peral, director de la parte alcalaína del proyecto Auger y Profesor Titular de Física Aplicada, y Dolores Rodríguez Frías, Profesora Titular de Física Atómica, Molecular y Nuclear, como profesores numerarios de la UAH, colaboran en este importante proyecto internacional. 
Asimismo el grupo, cuenta con la participación de un Profesor Asociado, Raúl Gómez Herrero, María Monasor Denia, becaria FPI de la Junta Castilla-La Mancha y Germán Ros Magán, becario FPI de la Comunidad de Madrid.

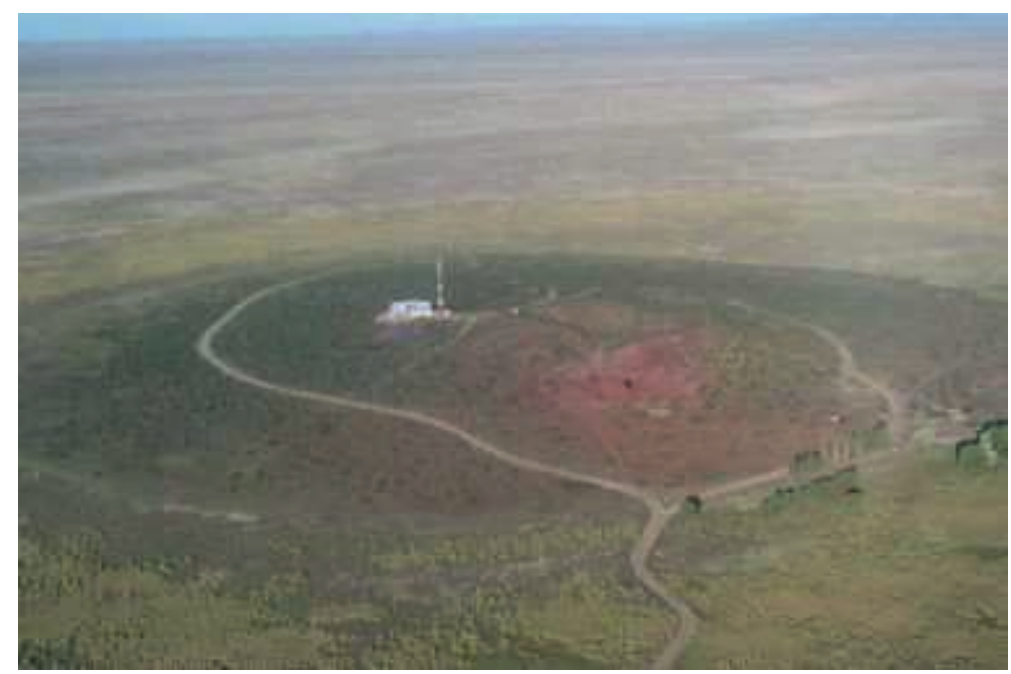

Vista aérea del edificio central del observatorio

Hasta la fecha, el trabajo de estos científicos ha consistido en el montaje de equipos e infraestructuras al pie de los Andes, en el altiplano de la Pampa argentina, concretamente en el pueblecito de Malargüe (provincia de Mendoza). El trabajo no ha sido fácil. Simplemente llegar hasta allí representa, además de las horas de vuelo hasta Mendoza, más de cinco horas de automóvil por desérticos páramos, vadeando ríos (a veces con problemas para sacar el coche del barro) y sin posibilidad de comunicaciones.

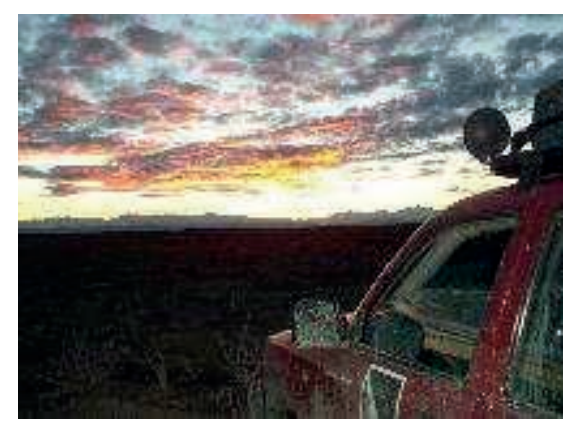

Puesta de sol en el camino 


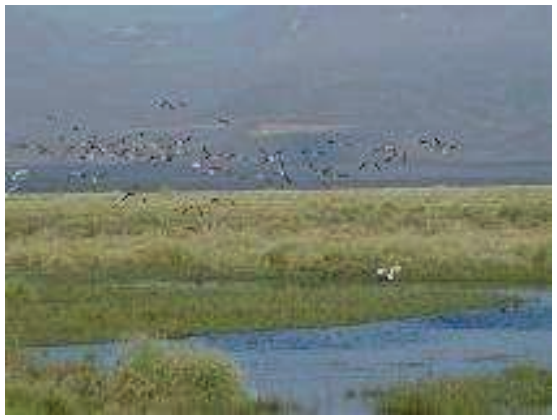

Flamencos en el camino

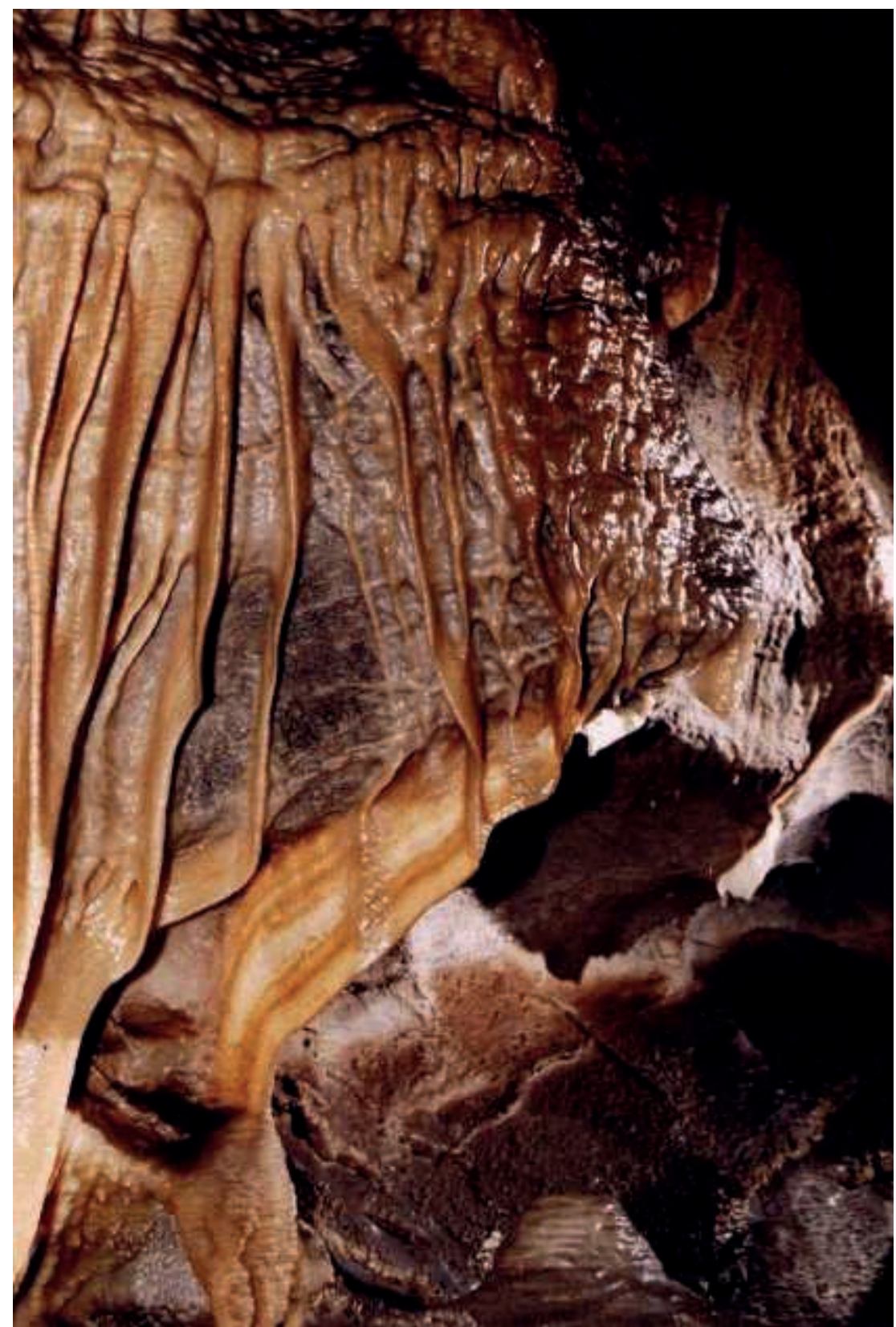


Interior de una caverna en Malargüe

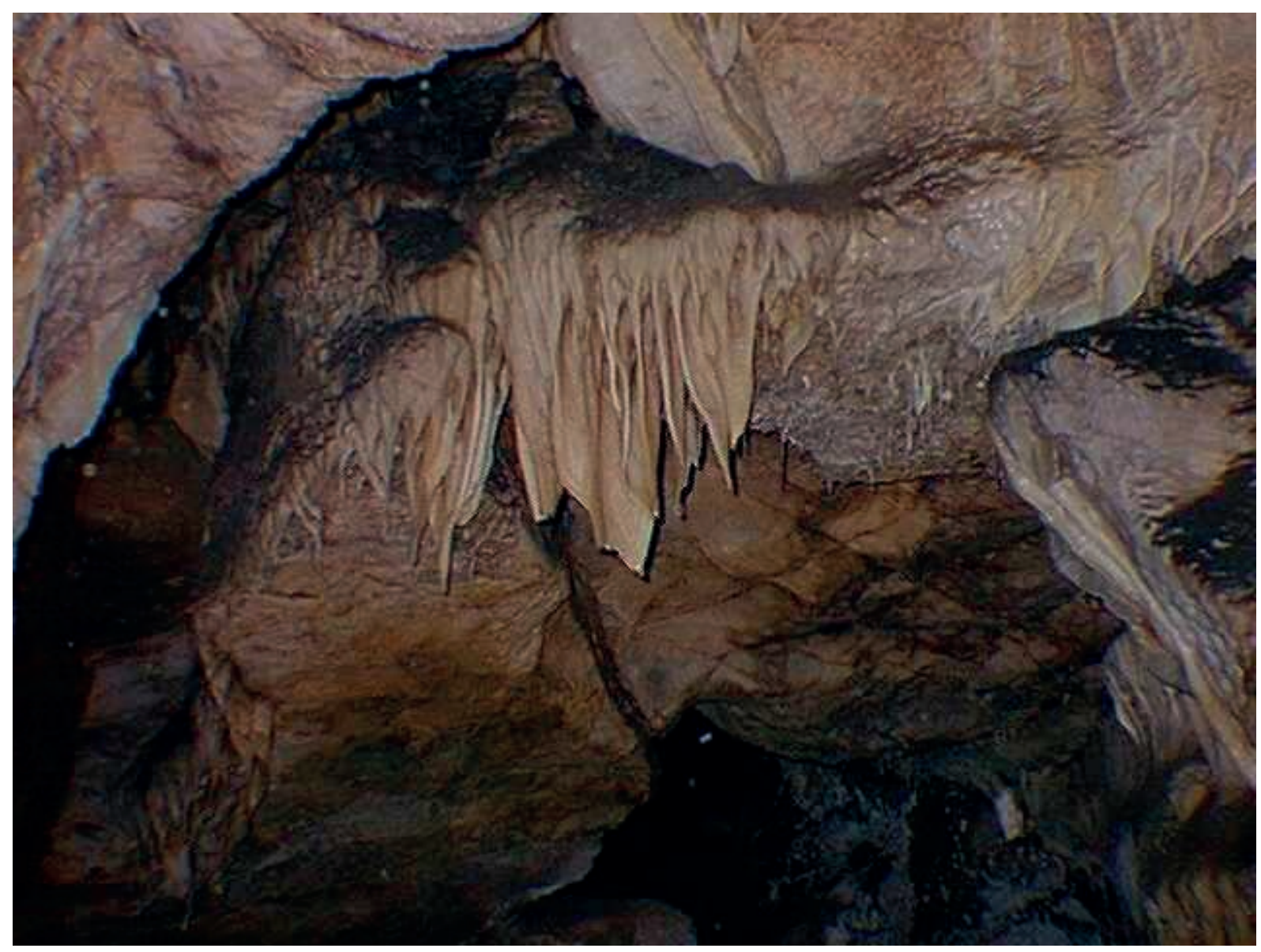

Otra vista del interior de la caverna 


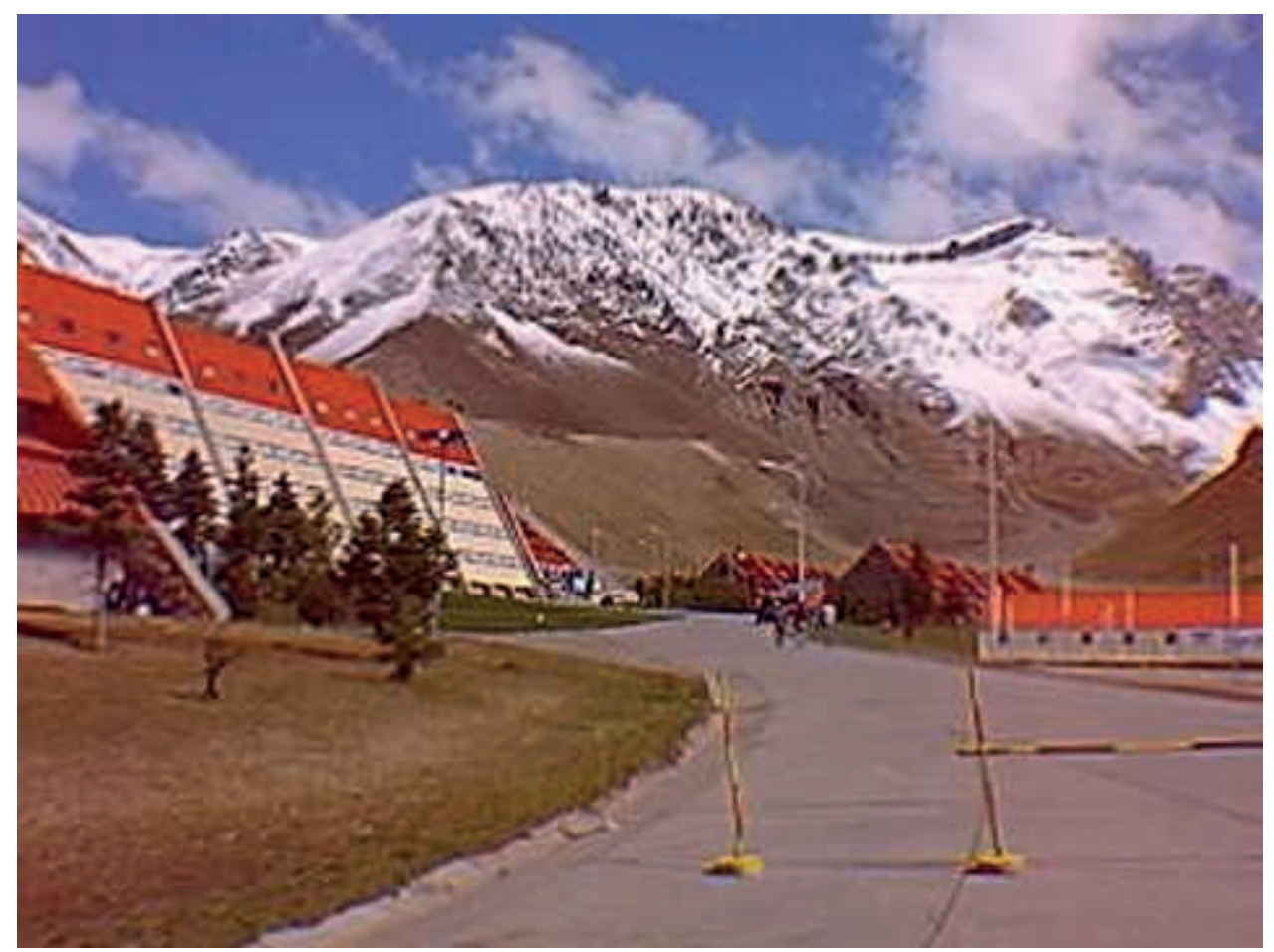

Vista de los Andes

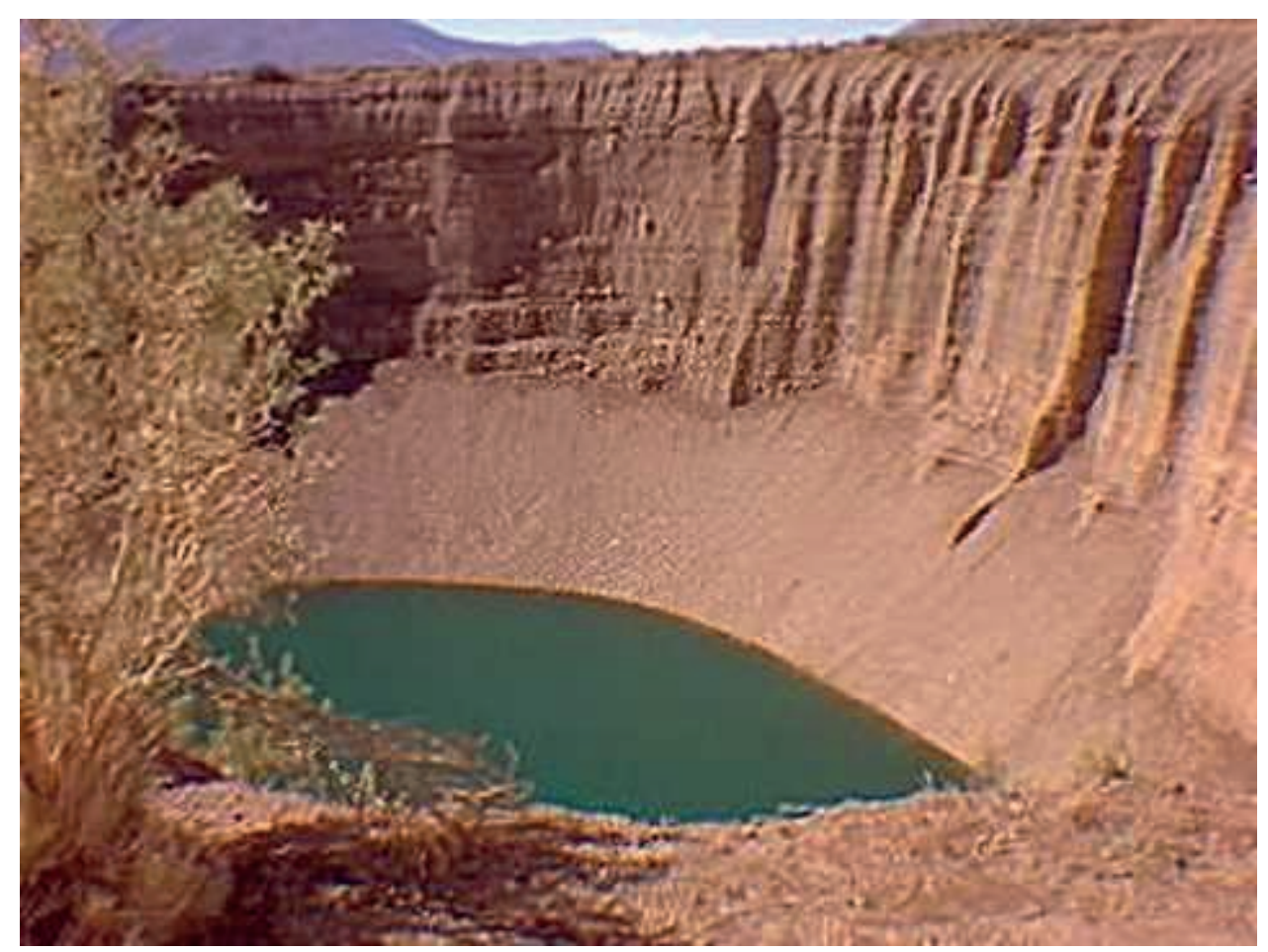

Una bonita laguna, no lejos del observatorio 
Del 9 al 11 de noviembre se inauguró la primera fase del experimento, el Observatorio del Hemisferio Sur, pues para cubrir la totalidad de la esfera celeste, se necesitan dos observatorios, uno en el Hemisferio Sur, el recién inaugurado, y otro en el Hemisferio Norte, que se comenzará a construir el año próximo en el Estado de Colorado (EE.UU.)

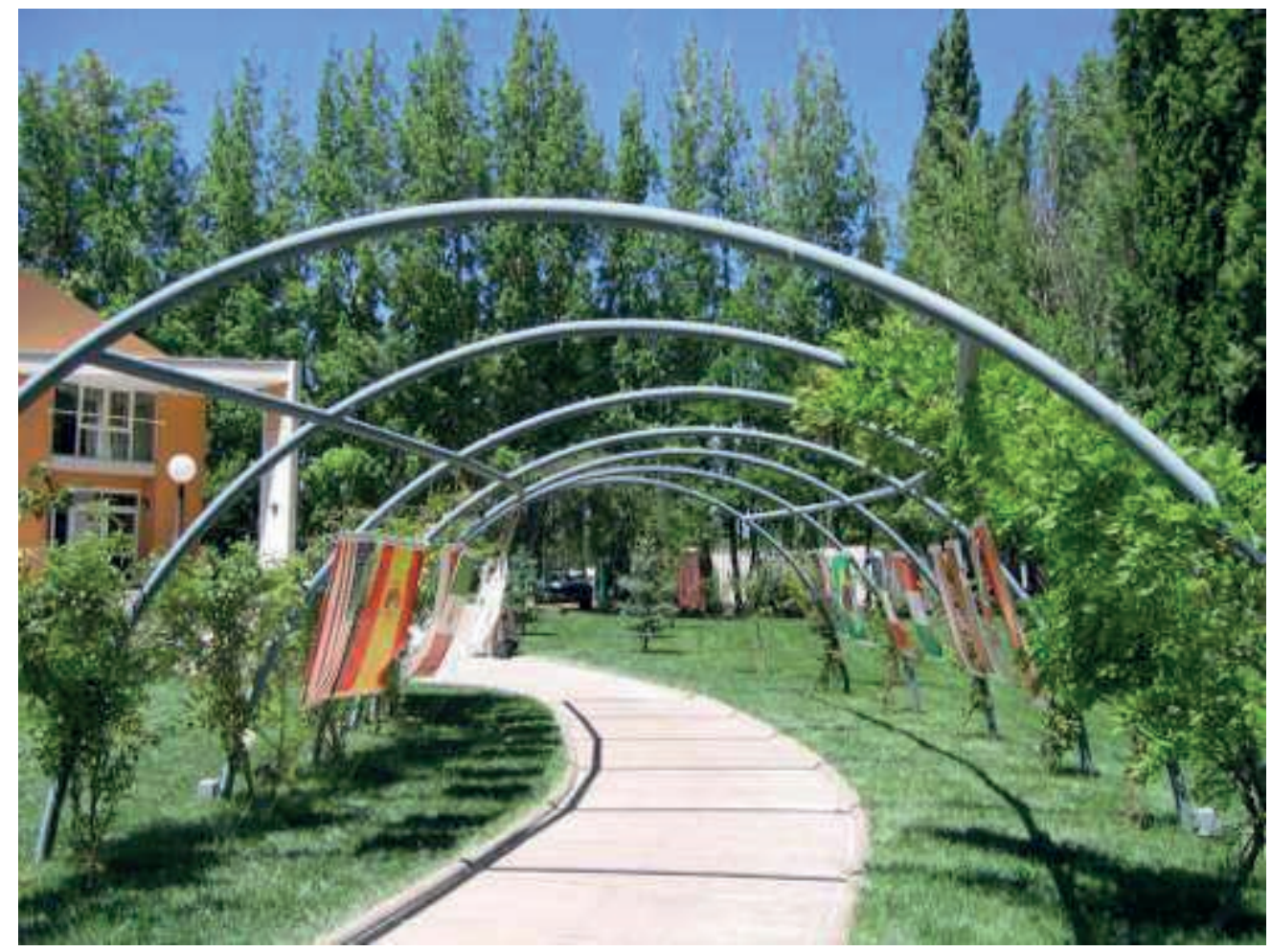

Vista del centro momentos antes de la inauguración 


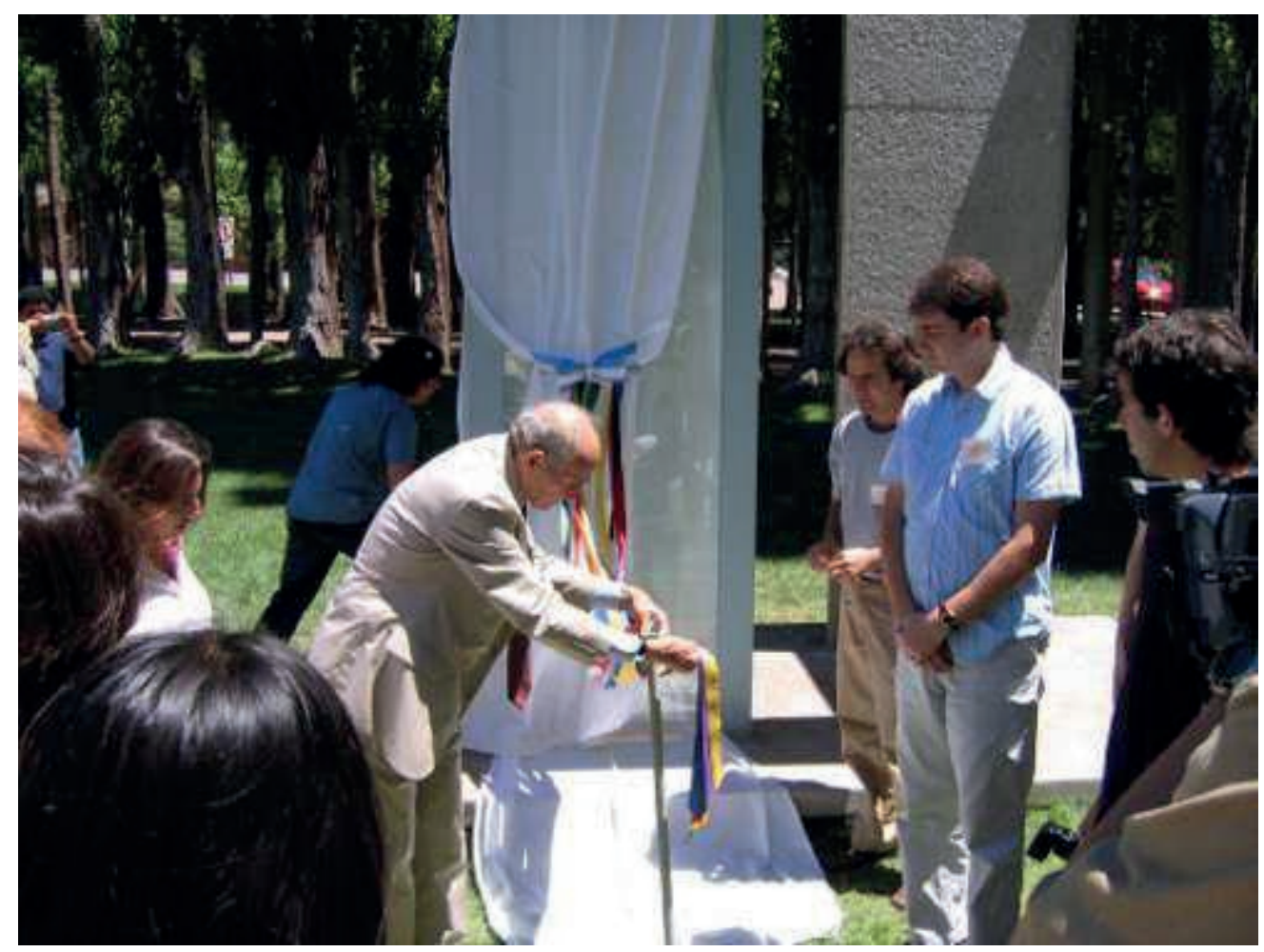

El premio Nobel, Jim Cronin, corta las cintas que representan la colaboración de los diversos países

El Observatorio Sur consiste en una matriz de 1.600 detectores de radiación cósmica secundaria repartidos en una superficie plana de 3.000 kilómetros cuadrados, y cuatro telescopios de fluorescencia atmosférica.

Para que el lector se haga una idea del funcionamiento de los equipos, explicaremos someramente en qué consiste la radiación cósmica y cómo se detecta.

Los rayos cósmicos consisten en partículas provenientes de fuera de nuestra atmósfera, desde todas las direcciones, con diversa composición (desde protones, es decir núcleos de hidrógeno, a iones de hierro, pasando por radiación electromagnética) y diferente origen (desde el Sol hasta las galaxias más lejanas). En este experimento se detectarán las partículas más energéticas conocidas de la radiación cósmica, algunas de ellas poseen energía que supera en más de cien 
millones la energía hasta ahora conseguida con los aceleradores más modernos construidos y, por tanto, viajan a velocidades muy próximas a la velocidad de la luz en el vacío; muy posiblemente esas energías tan elevadas jamás podrán conseguirse en un laboratorio terrestre. Por esta razón, el observatorio Pierre Auger constituye un laboratorio natural único, donde podemos poner a prueba las teorías nucleares y de composición del Universo actualmente admitidas.

Nadie conoce las fuentes de estos rayos cósmicos ultra-energéticos. La mayoría de las partículas de rayos cósmicos de baja energía que llegan a la Tierra provienen de nuestra propia galaxia, la Vía Láctea. Muchos provienen de la explosión de estrellas llamadas supernovas. Sin embargo, las partículas ultra-energéticas, probablemente, provengan de fuentes fuera de la vía Láctea, pero ¿de dónde?

No conocemos ninguna fuente en el Cosmos que pueda producir partículas con estas energías, ni siquiera en las más violentas explosiones de estrellas. Su origen oculta el secreto del inicio y la evolución del Universo. Algo en el cosmos está lanzando partículas a energías increíbles por el universo. ¿De dónde provienen estas partículas? ¿De alguna explosión cósmica super-poderosa? ¿De un enorme agujero negro que absorbe estrellas en una muerte violenta? ¿De colisiones entre galaxias? ¿Del colapso de residuos masivos procedentes del origen del universo?

Afortunadamente, esas partículas no llegan hasta la superficie terrestre, lo que haría inviable la vida tal como la conocemos, y son paradas por la alta atmósfera -a unos 37 Km de altura-, gracias a la interacción con las moléculas que componen el aire que respiramos. Estas interacciones provocan la aparición de nuevas partículas, menos energéticas, que sí llegan a la Tierra, producidas en una auténtica avalancha (cascada), y se pueden detectar directamente. 
Evidentemente se podrían detectar esas partículas primarias ultra-energéticas directamente, mediante satélites en órbita terrestre. No obstante, su flujo es muy pequeño y sería una casualidad demasiado eventual conseguir detectar una en cualquiera de tales instrumentos orbitales. Por ello, lo mejor es repartir muchos detectores por una gran superficie a nivel del suelo y medir las cascadas de las partículas secundarias, producto de la interacción atmosférica. Eso es lo que hace el Observatorio Pierre Auger, distribuyendo una red de 1.600 detectores, distanciados a 1,5 km entre sí y cubriendo una superficie total de $3.000 \mathrm{~km} 2$.

Estos detectores no son otra cosa que tanques llenos de agua muy pura que observan la luz emitida por las partículas de la cascada al moverse por el líquido. Esa luz es muy tenue, por lo que debe ser amplificada antes de medir cualquiera de sus características.

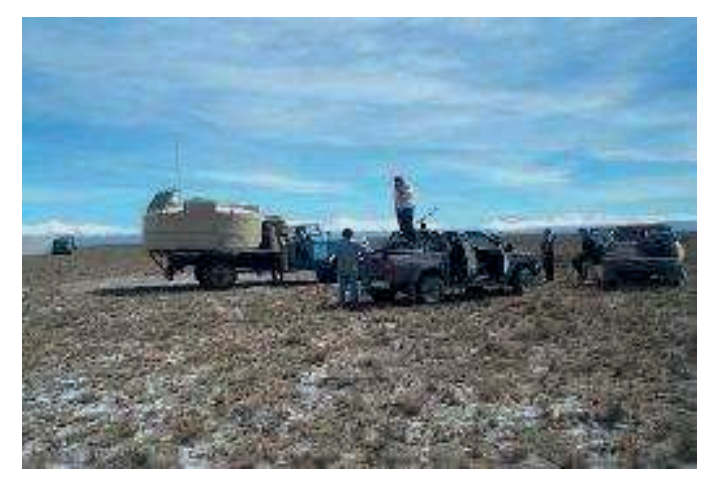

Instalando uno de los tanques

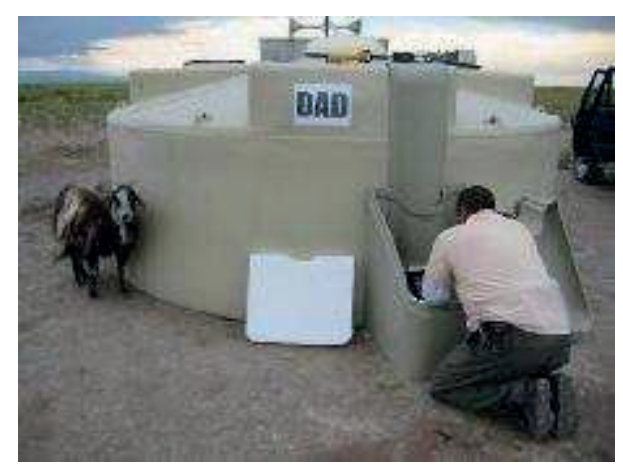

Cada uno lleva el nombre de un niño del pueblo de Malargüe 
El complejo Pierre Auger, no obstante, es un observatorio mixto, pues también observará la entrada de las partículas ultra-energéticas en la alta atmósfera, mediante una serie de telescopios de alta sensibilidad que, en las noches despejadas sin Luna, escudriñará la atmósfera para observar la tenue luz ultravioleta que producen las cascadas de rayos cósmicos al atravesar el aire, pudiendo así determinar el origen de la interacción.

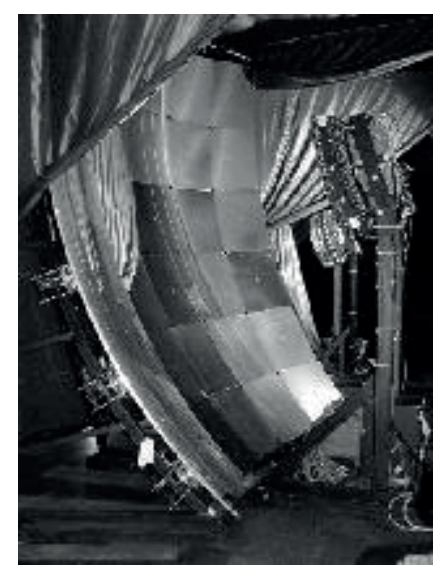

Vista de uno de los espejos de los telescopios de fluorescencia

El Observatorio Pierre Auger será el mayor dedicado a la detección y estudio de los rayos cósmicos jamás construido, y abrirá una nueva ventana al conocimiento del Universo, llegando hasta donde nunca antes se había alcanzado. A partir de ahora, los datos experimentales obtenidos aportarán información sobre el origen del Universo, permitirán contrastar las teorías de Einstein y resolverán incógnitas sobre la materia y energía oscuras, predichas por las teorías cosmológicas modernas y aún no detectadas. 